Methods An initial list of items has been developed based in a systematic review done for our team about the guidance in updating handbooks. The working group has reviewed the initial list and reached consensus about the items to include. Through a survey, we will present the initial list to a multidisciplinary group of international experts and we will ask them about what is the relevant information that needs to be reported in an updated CPG, and about the elements to be included in a high-quality updating process.

Results These results will give us insight in what elements are required to be reported an updated CPG. Additionally, we will gain information about what kind of elements include a highquality updating process.

Discussion and Implications This checklist will help people responsible for updating CPGs, in conducting and reporting their update in a high-quality manner. Ultimately this might results in more up-to-date recommendations and more valid CPGs.

\section{P331 EVALUATION OF QUALITY OF CZECH NEUROLOGY CLINICAL PRACTICE GUIDELINES USING AGREE II INSTRUMENT}

${ }^{1,2} \mathrm{R}$ Licenik, ${ }^{1} \mathrm{~K}$ Klikova, ${ }^{1} \mathrm{D}$ Osinova, ${ }^{1,2} \mathrm{~S}$ Doubravska, ${ }^{1} \mathrm{~K}$ Ivanova. ${ }^{1}$ Centre for Clinical Practice Guidelines, Faculty of Medicine, Palacky University, Olomouc, Czech Republic; ${ }^{2}$ Northwick Park Hospital, Hyper Acute Stroke Unit, London, United Kingdom

10:1136/bmjgs-2013-002293.260

Background The quality of guidelines has been variable. Evidence-based clinical practice guidelines should be of the highest possible quality, which has to be systematically evaluated. Obj TRiaDS, Research Methodology Group.

Objectives We have evaluated quality of new set of CPGs developed by Czech neurological society between 2009 and 2011 using AGREE II instrument.

Methods Six CPGs developed by Czech neurology society were assessed using AGREE II instrument by 4 independent assessors. Uncertainties and missing information were solved by the authors. Expert opinion on the evaluation process and outcomes were obtained.

Results All evaluated CPGs were of high quality with average of 93.5\% for total score domain. The CPGs had high scores in the Clarity and recommendations and Applicability (99.6\%), Scope (99.0\%), Rigour of development (97.6\%) and Stakeholder involvement (97.1\%). The lowest score was in Editorial independence $(47.5 \%)$.

Discussion The overall quality of neurology CPGs was high because of the methodology and support for developers was robust, systematic and standardised. Only the editorial independence scored low. The statement of conflict of interest is missing in all the CPGS as it is not a part of the methodology guidelines. Implications for Guideline Developers/Users High quality CPGs can be developed even in a small country with limited budget if the methods of development are well designed, implemented and evaluated.

\section{P334 CHALLENGES AND ADVANTAGES ADAPTING RECOMMENDATIONS: GPC BREAST CANCER}

${ }^{1} \mathrm{E}$ Peña Torres, ${ }^{2} \mathrm{M}$ Alba, ${ }^{3} \mathrm{~F}$ Sierra. ${ }^{1}$ Instituto Nacional de Cancerología, Bogotá DC, Colombia; Instituto Nacional de Cancerología, Bogotá DC, Colombia; ${ }^{3} / n s t i t u t o$ Nacional de Cancerología, Bogotá DC, Colombia

10:1136/bmjqs-2013-002293.261
Background Development of a high-quality Clinical Practice Guideline (CPG) involves spending a significant amount of resources. Frequently, similar strategies are used around the world to achieve similar results. A method to utilise resources in a more efficient manner and to avoid the unnecessary duplication of efforts is to adapt existing guidelines. However, the methodological diversity of CPGs, specific regional conditions, and difficulties in the implementation of recommendations, impose a systematic approach for its adaptation that constitute an important methodological challenge, that must take into account the similarities between a de novo development and an adaptation of guidelines. The process of adaptation requires systematic and transparent activities that disclose the considerations made to adjust the recommendations to a specific context.

Methods The development of the GPC for breast cancer follows the steps proposed in the Methodological Guideline for the development of GPCs in the Colombian Health System.

Results The systematic search retributed 176 GPCs, 48 (27\%) of which were qualified with the DELBI instrument as "highly recommended". The Guideline Development Group (GDG) elaborated 41 questions, 30 (73\%) were adapted following the methodology proposed by the ADAPTE collaboration. The remaining 11 (27\%) developed de novo; 12,000 titles and publication summaries have been reviewed, of which 130 have been included and qualified with the system proposed for GRADE Working Group. The results take into account the challenges implied in the CPG adaptation processes.

\section{P340 SOURCES OF INFORMATION FOR DEVELOPMENT OF CLINICAL PRACTICE GUIDELINES IN JAPAN}

${ }^{1,2} \mathrm{Y}$ Hatakeyama, ${ }^{1,3} \mathrm{~A}$ Okumura, ${ }^{1} \mathrm{~N}$ Takahashi, ${ }^{1,4} \mathrm{M}$ Yoshida, ${ }^{1,5} \mathrm{~N}$ Kojimahara, ${ }^{1,5} \mathrm{~K}$ Kiyohara, ' ${ }^{1,5} \mathrm{Y}^{\mathrm{S}}$ Sato, ${ }^{1,6} \mathrm{~N}$ Htun, ${ }^{1} \mathrm{MINDS}$ Group, ${ }^{1,5} \mathrm{~N}$ Yamaguchi. ${ }^{1} \mathrm{MINDS}$ Center, EBM and Guidelines Division, Japan Council for Quality Health Care, Tokyo, Japan; ${ }^{2} T$ The University of Tokyo, Tokyo, Japan; ${ }^{3}$ Department of Social Medicine, The University of Tokyo, Tokyo, Japan; ${ }^{4}$ International University of Health and Welfare, Tokyo, Japan; ${ }^{5}$ Department of Public Health, Tokyo Women Medical University, Tokyo, Japan; ${ }^{6}$ Department of Molecular Epidemiology, Tokyo Medical and Dental University, Tokyo, Japan

\section{0:1136/bmjqs-2013-002293.262}

Background A Systematic search for literature is the basis of systematic review for development of trustworthy clinical practice guidelines (CPGs). Recently, standard method of literature search has been established. However, it is not clear what sources of information are used for development of CPGs in Japan.

Objectives The aim is to clarify what sources of information are used for development of CPGs in Japan.

Methods We reviewed Japanese CPGs that have been selected by MINDS (Medical Information Network Distribution Service) through process of screening and evaluation in 2011-2013, and abstracted description about sources of information used in these CPGs in February 2012.

Results A total of 88 Japanese CPGs selected by MINDS in 2011-2013. 65 (73.9\%) CPGs used Medline, 29 (33.0\%) used Cochrane database of systematic reviews, and 51 (58.0\%) used Ichushi. Related CPGs was used in 30 (34.1\%) CPGs. Hand search was used in $56(63.6 \%)$ CPGs. There was no clear description of source of information in 18 (20.5\%) CPGs.

Discussion Medline was used in most CPGs, and Cochrane database of systematic reviews was used in a relatively small number of CPGs. Ichushi, which is a database of domestic medical 
literature information in Japan, was used in about a half of Japanese CPGs.

Implications for Guideline Developers/Users It is necessary for further study to clarify what sources of information should be used for development of trustworthy CPGs.

\section{P342 ASSESSMENT OF THE RECOMMENDATIONS STRUCTURE IN EIGHT CLINICAL PRACTICE GUIDELINES DEVELOPED IN COLOMBIA}

1,2 1 Florez, ${ }^{1} \mathrm{~A}$ Perez, ${ }^{1} \mathrm{~L}$ Prieto, ${ }^{1} \mathrm{~L}$ Cañon. ${ }^{1}$ Instituto de Evaluación Tecnológica en SaludIETS, Bogota, Colombia; ${ }^{2}$ Universidad de Antioquia, Medellin, Colombia

\section{0:1136/bmjqs-2013-002293.263}

Background There is not enough information to guide editorial wording of recommendations within CPG (Clinical Practice Guidelines) development. The AGREE-II instrument suggests the inclusion of population, intervention and outcome (P-I-O) components within recommendations.

Objective To evaluate P-I-O component in CPG recommendations and to analyse its relationship with the AGREE-II evaluation.

Methods Eight recently developed in Colombia CPG were chosen and assessed by four methodological experts; the presence of P-I-O component in each recommendation was established, and compared with an external evaluation score of the 15th item of AGREE-II instrument.

Results Eight guidelines with a total of 691 recommendations were evaluated, all of them were appraised by external international review with the Spanish AGREE-II instrument and its use were recommended. An average of $9.9 \%$ of recommendations met P-I-O structure; the absence of each component was 31.2\% for population, $6 \%$ for intervention and $85.2 \%$ had no outcome. The item 15 of AGREE-II instrument reported results between 4 and 7 , scores of good quality.

Discussion Recommendations in CPG seem to be clear, but most of them don't contain the evaluated criteria for their report. External evaluation emphasises in clarity of recommendation, and there is no agreement with the percentages obtained according to P-I-O structure, which enhance its understanding. Implications It is advisable to standardise methodology for recommendations to include all components that reflect the answer to the research question.

\section{P343 SELLING OLD WINE IN NEW BOTTLES: WHY IT IS WORTHWHILE TO HAVE ANOTHER GUIDELINE DEVELOPMENT HANDBOOK?}

C Muche-Borowski, M Nothacker, I Kopp. AWMF Institute of Medical Knowledge Management, Marburg, Germany

\section{0:1136/bmjqs-2013-002293.264}

Background Since 1995, the Association of the Scientific Medical Societies in Germany (AWMF) maintains an open access, quality assured guideline register (www.awmf.org), currently containing 676 guidelines developed by 168 societies. While the societies are responsible for topic selection, development and content, the mission of AWMF is to promote, support and coordinate guideline development and to ensure the overall quality of the guideline register. To achieve this goal, AWMF established a new set of recommendations and rules.
Context Published manuals for guideline developers do not fully meet the requirements of our national guideline system. Our manual takes into account the - development of methodological strategies - international consensus on key criteria for "good guidelines" - target group including guideline novices and clinical experts wishing to be informed about methodological developments but to delegate project management and basic methodological work - informational need to include guidelines into the AWMF register - specific requirements of the German health care system (e.g. the patients' rights act)

Description of Best Practice Our manual consists of two parts. The first part includes recommendations and practical advice for guideline developers. The second part includes rules AWMF applies to judge inclusion into the register (e.g. addressing the management of conflict of interests).

Lessons for Guideline Developers, Adaptors, Implementers, and/ or Users Our guideline development handbook which will be updated continuously may serve as an example for methodological guidance that is based on a systematic review of the international literature as well as the identification of national requirements and experience.

\section{P348 PATIENT AND CONSUMER INVOLVEMENT IN GUIDELINE INTERPRETATION AND PATIENT TOOL DEVELOPMENT; A COMPARISON OF TWO FOCUS GROUPS AND UNDERSTANDING CULTURAL DIVERSITY}

${ }^{1} \mathrm{C}$ Davino-Ramaya, ${ }^{2} \mathrm{~K}$ Ramaya, ${ }^{1} \mathrm{H}$ Tucker. ${ }^{1}$ Kaiser Permanente (Northwest Permanente), Portland, USA; ${ }^{2}$ Pacific University, Forest Grove, USA

\section{0:1136/bmjqs-2013-002293.265}

Incorporating patient and consumer involvement in clinical practice guideline (CPG) activities has become a priority for health care organisations internationally. In diverse populations with potential healthcare disparities the development of culturally competent patient tools based on interpretation of preexisting clinical practice guidelines is also considered important practice for many organisations that adopt external guidelines. In our exploration of the role of consumer engagement in the development of CPG support tools and in addressing healthcare disparities we compare the results of two culturally different groups through the evaluation of pre and post surveys as well as in-person focus groups. Survey intent was to gauge awareness of the existence of CPGs and gauge interest in developing patient and consumer support tools. Results of one culturally diverse group are compared with a minority group. We analyse the perceptions and attitudes of high priority health care issues identified by both groups. Our findings underscore the opportunity for health care organisations with significantly different populations to address healthcare disparities in the development of patient and consumer CPG support tools. As an extension of our previous work on "collaborative engagement" our comparative results emphasise the need to appropriately address cultural, language and health literacy issues as well as addressing health disparities between populations. We focus on identifying barriers to access and treatment and discuss implication for practice as well as future directions.

\section{P352 GAME-IT (GAMES FOR IMPROVING TREATMENT- RECOMMENDATIONS)}

${ }^{1} \mathrm{~L}$ Brandt, ${ }^{2} \mathrm{~S}$ McCallum, ${ }^{1} \mathrm{~A}$ Kristiansen, ${ }^{3} \mathrm{~T}$ Agoritsas, ${ }^{4} \mathrm{E}$ Akl, ${ }^{1} \mathrm{P}$ Vandvik, ${ }^{5} \mathrm{~V}$ Montori. ${ }^{1}$ Department of Medicine, Innlandet Hospital Trust, Gjøvik, Norway; ${ }^{2}$ Game 